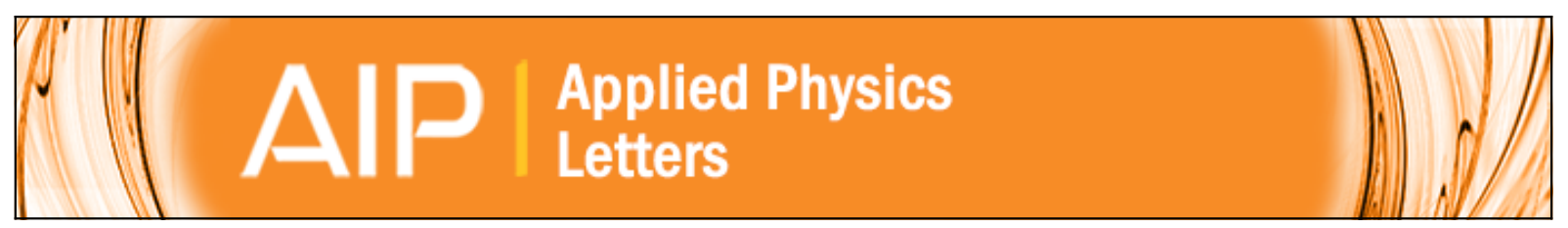

\title{
Plasmonic crystal enhanced refractive index sensing
}

Benedikt Stein, Eloïse Devaux, Cyriaque Genet, and Thomas W. Ebbesen

Citation: Applied Physics Letters 104, 251111 (2014); doi: 10.1063/1.4885120

View online: http://dx.doi.org/10.1063/1.4885120

View Table of Contents: http://scitation.aip.org/content/aip/journal/apl/104/25?ver=pdfcov

Published by the AIP Publishing

\section{Articles you may be interested in}

Refractive index dispersion sensing using an array of photonic crystal resonant reflectors

Appl. Phys. Lett. 107, 061101 (2015); 10.1063/1.4928548

Refractive index sensor based on hybrid coupler with short-range surface plasmon polariton and dielectric waveguide

Appl. Phys. Lett. 100, 111108 (2012); 10.1063/1.3693408

Silicon photonic crystal nanostructures for refractive index sensing

Appl. Phys. Lett. 93, 181103 (2008); 10.1063/1.3009203

Surface plasmon enhanced diffraction in cholesteric liquid crystals

Appl. Phys. Lett. 90, 183115 (2007); 10.1063/1.2735957

Digital-holography refractive-index-profile measurement of phase gratings

Appl. Phys. Lett. 88, 111114 (2006); 10.1063/1.2186515

\section{Precise temperature control for cryogenic research}

Model 372

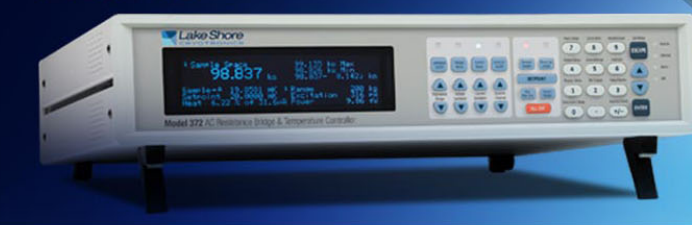




\title{
Plasmonic crystal enhanced refractive index sensing
}

\author{
Benedikt Stein, Eloïse Devaux, Cyriaque Genet, ${ }^{a}$ and Thomas W. Ebbesen \\ ISIS \& icRFC, University of Strasbourg and CNRS, 8 allée Gaspard Monge, 67000 Strasbourg, France
}

(Received 21 April 2014; accepted 12 June 2014; published online 25 June 2014)

\begin{abstract}
We demonstrate experimentally how the local anisotropy of the dispersion relation of surface plasmon modes propagating over periodic metal gratings can lead to an enhancement of the figure of merit of refractive index sensors. Exploiting the possibility to acquire defocused images of the Fourier space of a highly stable leakage radiation microscope, we report a twofold increase in sensing sensitivity close to the band gap of a one-dimensional plasmonic crystal where the anisotropy of the band structure is the most important. A practical sensing resolution of $O\left(10^{-6}\right)$ refractive index units is demonstrated. (C) 2014 AIP Publishing LLC.

[http://dx.doi.org/10.1063/1.4885120]
\end{abstract}

Surface plasmon (SP)-based sensors have become standard systems in the detection of ultra small refractive index changes, and are thus routinely used today in a great variety of contexts, from analytical chemistry to medicine. ${ }^{1,2}$ This stems from the fact that SP sensors offer a unique combination of capacities: high-throughput, real-time monitoring, and label-free characterization at the level of single molecular interactions, all this within micron-scaled volumes of interactions. ${ }^{3,4}$ The efficiency of plasmonic sensing has naturally been accompanied by a burst of recent work with specific SP-based architectures all aiming at improving sensitivity and resolution (see Refs. 5-7 and references therein).

In this context, we have recently demonstrated a large improvement over the optimal figure of merit (FOM) of an SP-based sensor by monitoring the evolution, as a function of the refractive index change $\delta n$, of the normalized intensity variation $\delta i(k)$ of a SP resonant profile, imaged in the Fourier $k$-plane. The SP mode being characterized by its wave vector $k_{\mathrm{SP}}$, the sensitivity $S$ of the sensor is defined by

$$
S=\left|\frac{\delta i(k)}{\delta n}\right|=\left|\frac{\delta i(k)}{\delta k}\right| \times\left|\frac{\delta \operatorname{Re}\left[k_{\mathrm{SP}}\right]}{\delta n}\right| .
$$

This expression immediately reveals two different possible strategies to increase the sensitivity: either by optimizing $\delta i(k) / \delta k$ or $\delta \operatorname{Re}\left[k_{\mathrm{SP}}\right] / \delta n$. Our recent work optimized the former factor exploiting the built-in asymmetry of a Fano resonance recorded on an intensity interrogation scheme developed on an advanced leakage radiation microscope (LRM). ${ }^{8}$

In this Letter, we aim at tailoring the value of the second factor $\delta \operatorname{Re}\left[k_{\mathrm{SP}}\right] / \delta n$, exploiting a band gap effect in a plasmonic crystal, as proposed theoretically recently by us ${ }^{10}$ and others in a different context. ${ }^{9}$ While $\delta \operatorname{Re}\left[k_{\mathrm{SP}}\right] / \delta n$ is a constant for SP modes propagating along planar interfaces due to the associated isotropic SP dispersion relation $\omega\left(\operatorname{Re}\left[k_{\mathrm{SP}}\right]\right)$, the situation turns out to be different on plasmonic gratings where the dispersion relation becomes anisotropic. Basing our demonstration on the same LRM imaging setup to record ultra-stable Fourier space images, we show how this

\footnotetext{
a)Electronic mail: genet@unistra.fr
}

anisotropy can be exploited for high-resolution refractive index sensing, giving the possibility to control and thus amplify the global sensitivity of the sensor.

Our sensor geometry is shown schematically in Fig. 1(b). A nano groove source is illuminated by a laser spot to launch SPs propagating inside a singly periodic sinusoidal metal grating (1PG). We recall that for a grating period chosen close to the Bragg condition, ${ }^{10}$ band gaps around the M-point of the grating appear, as clearly seen from the corresponding isofrequency surface (IFS) shown in Figs. 2(a). The effect of the anisotropy of the SP dispersion relation with respect to a refractive index change is simulated in Fig. 2(b) and compared to the response on a flat metal film using, in a wave vector interrogation scheme, the $x$ component $k_{x}^{\mathrm{SP}}$ of $\operatorname{Re}\left[k_{\mathrm{SP}}\right]$ as a sensing meter at a fixed $k$ position. For a flat metal film, a small $\delta n$ change shifts $k_{x}^{\mathrm{SP}}$ by $\delta k_{x}^{\text {flat }}$ determined from the SP dispersion relation. It is immediately clear that over the plasmonic crystal, the anisotropic band structure will act as a lever on the detected wave vector change, with an enhanced $\delta k_{x}^{1 \mathrm{PG}}$ change. Such levering results in an equivalent increase of the sensor sensitivity, defined as $S_{k}=$ $\delta k_{x}^{\mathrm{SP}} / \delta n$ in the same wave vector interrogation scheme. Close to the Bragg condition $\left(k_{y} \sim 0.25 \mu \mathrm{m}^{-1}\right)$, a large increase of the sensitivity will be observed, stemming from the diverging slope of the SP band at this M-point.

The performance of the sensor can be assessed by choosing a standard definition of the figure of merit with $\mathrm{FOM}=S_{k} / w$ where $w$ is the full width at half maximum of the dip. At a flat interface, this width is fixed by SP loss according to $w_{\text {flat }}=1 / 2 \operatorname{Im}\left[k_{\mathrm{SP}}\right]$. Assuming the same loss on the plasmonic crystal, it is important to stress that the width at an arbitrary point of the IFS of the grating will broaden due to a geometric increase of the cross-section as $w_{1 \mathrm{PG}}=w_{\text {flat }} / \cos \left[\arctan \left(\delta k_{x}^{\mathrm{SP}} / \delta k_{y}\right)\right]$. Such a correction still yields for our setup an evaluated FOM improvement for the 1PG sensor of $36 \%$ for an optimal choice of $k_{y}$.

To validate this approach, we specifically designed a LRM setup performing as a high-resolution sensor. We monitored, as a function of refractive index changes, the evolution of the anisotropic SP dispersion relation at the level of a smooth one dimensional metal grating used as a plasmonic crystal. We imprinted a $1 \mathrm{PG}$ directly on an indium-tin oxide substrate using focused ion beam (FIB) lithography on which 

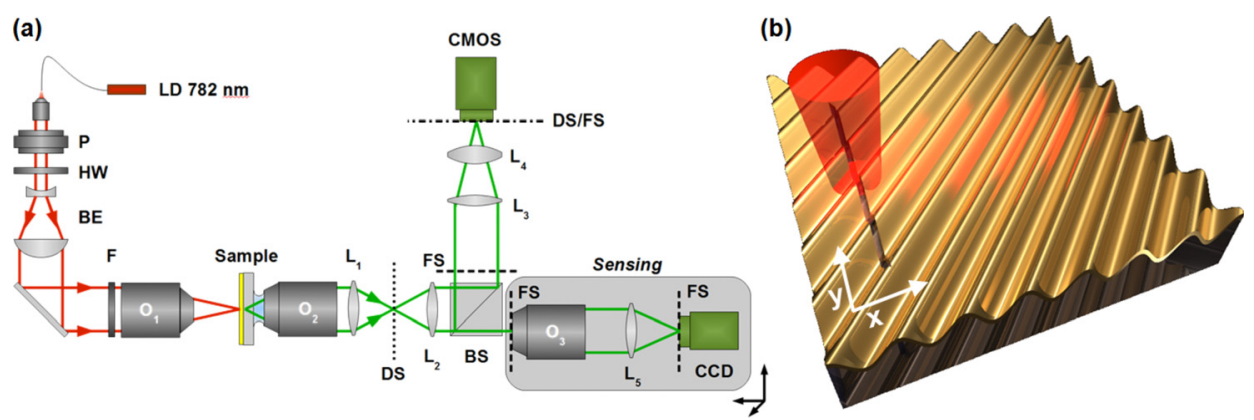

FIG. 1. (a) Optical setup: light from a $\lambda_{\mathrm{L}}=782 \mathrm{~nm}$ pigtailed laser diode (LD) passing a polarizer (P1), half-wave plate (HW), beam expander (BE), is weakly focused by a microscope objective on the plasmonic crystal in a PDMS microfluidic cell. SP modes are decoupled by an NA $=1.49$ immersion objective and imaged by two lenses (L1, L2) to intermediate direct space (dotted line) and Fourier $k$-space (dashed line) planes, respectively. Spatial filtering of direct transmission is conveniently performed in the first intermediate direct space plane. The Fourier space can be scanned with high magnification by a third objective and recorded with a charge-coupled device (CCD) after an imaging lens (L5). Defocused images are recorded by controlled axial shifts of this whole unit, as illustrated by the gray box. (b) Sensing scheme: an SP mode is launched on a one-dimensional plasmonic crystal (1PG) from a nano groove source illuminated by monochromatic light and oriented $45^{\circ}$ with espect to the sinusoidal grating vector of the $1 \mathrm{PG}$.

a thin $(80 \mathrm{~nm})$ layer of gold was evaporated. Remarkably, the ionic lithography process reduces the indium-tin oxide initial roughness and we therefore obtained, after the metal deposition, a very smooth grating with a surface quality that would not have been achievable by milling directly the metal film. The design was carefully set with a period $P=580 / \sqrt{2}$ nm, i.e., close to the Bragg period evaluated for an SP mode at a gold-water interface where the SP wavelength $\lambda_{\mathrm{SP}}=2 \pi / \operatorname{Re}\left[k_{\mathrm{SP}}\right]=566 \mathrm{~nm}$ for an excitation laser wavelength $\lambda_{\mathrm{L}}=782 \mathrm{~nm}$ (see Ref. 10). The crystal was embedded in a PDMS microfluidic cell at the level of which the modifications of the fluidic environment of the sensor can be fully controlled with precision. ${ }^{11}$ Note that the thermal stability of the experimental environment was carefully monitored in order to avoid any temperature effect on the recorded signals.

Sensing was actually performed in the magnified Fourier space of the LRM, in a configuration described in Fig. 1(a). However, as it can be seen in Figs. 2(a), standard Fourier space leakage radiation images only provide a relatively low contrast at the level of the SP band gap, in which evolution turns out to be difficult to monitor precisely. To overcome this limitation, an original strategy is to exploit defocused images of the Fourier space. The resulting interference pattern turns out to be characterized by a well defined first diffraction dip, following approximately the shape of the SP band structure. It is this dip which is chosen as the refractive index probe. In order to record even more precisely its evolution, we further saturated the image at that minimum in order to reduce its width. A typical recorded image is shown in Fig. 3(a). Image cross-sections taken along $k_{y}$ at the band gap region are displayed in Fig. 3(b). It is important to realize that, because it is not possible to scale defocused images in terms of wave vector unit easily, the resonance shifts are all measured in pixel units (px).

Aiming at probing refractive index changes smaller than $10^{-5}$ RIU (refractive index units), deionized water solutions with varying concentrations of ethanol were prepared, scanning refractive index changes $\delta n=n_{\mathrm{sol}}-n_{\mathrm{H}_{2} \mathrm{O}}$ from $\delta n_{\min }=3.4 \times 10^{-6} \mathrm{RIU}$ (corresponding to $5.21 \times 10^{-5} \mathrm{w} / \mathrm{w}$
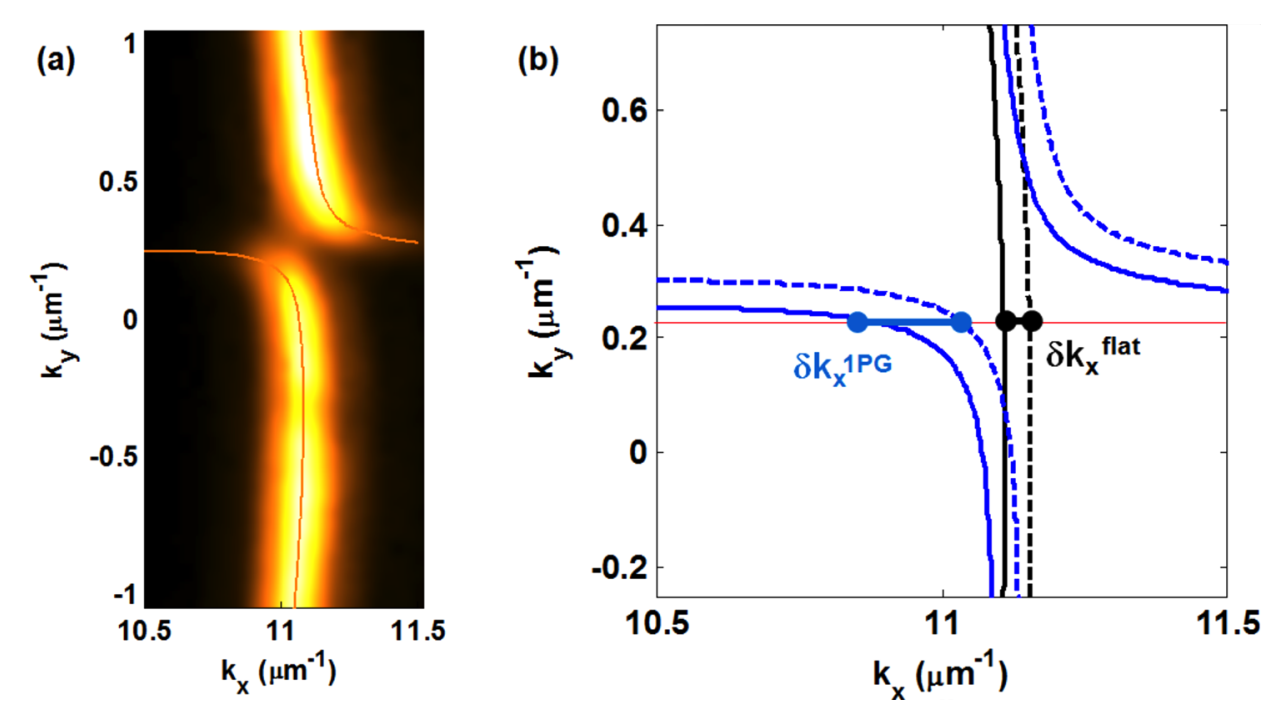

FIG. 2. (a) Zoomed image of the IFS around the M-point. The continuous lines are theoretical fits of the IFS fitting the 1 PG modulation depth. (b) 1 PG enhancement sensing scheme: on a flat gold film, the SP dispersion relation corresponds to a circular IFS (black continuous curve). A small change of the refractive index $\delta n$ leads to an equivalent increase of the circle radius (black dashed curve). On the 1PG, the SP dispersion relation displays an anisotropic IFS (blue continuous curve). The same $\delta n$ change now results in an amplified change $\delta k_{x}^{1 \mathrm{PG}}$ measured in the LRM Fourier image as a spatial shift $\Delta x_{\text {grat }}$ of the SP resonance enhanced with respect to the shift $\Delta x_{\text {flat }}$ on a flat film. 

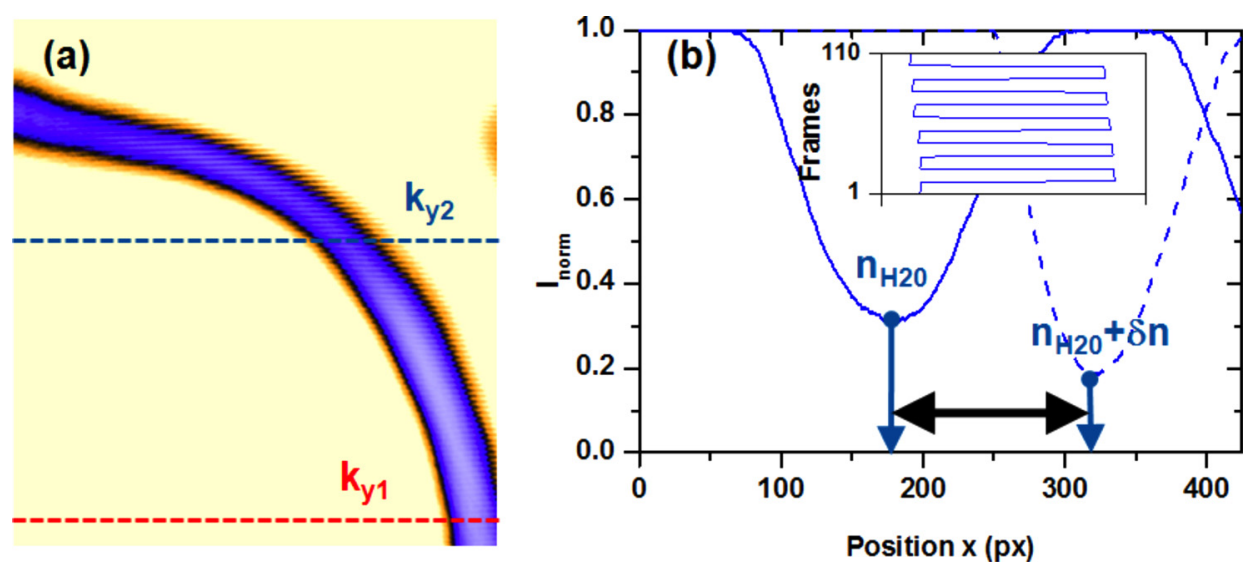

FIG. 3. (a) Magnified Fourier space leakage radiation image of the IFS close to the M-point recorded by slightly defocusing the magnification block around lens L5 with respect to the Fourier space conjugate plane (see Fig. 1(a)). The operating point close to the band gap is indicated by the blue line crossing the Fourier plane vertical axis at a value $k_{y 2}$. (b) Cross-sections along $k_{y 2}$ of the defocused leakage radiation image. These cross-sections are used for evaluating the sensitivity quantitatively. The profiles were taken in ultra-pure water (solution 1, continuous curve) and in a solution corresponding to a refractive index increase of $\delta n=4.3 \times 10^{-3}$ RIU (solution 2, dashed curve). Both cross-sections have been taken after a vertical spatial averaging over 17 lines of the CCD chip around the operating points shown in (a). The insert displays the variation of the detected dip centers $x_{j}$ given through iterative exchanges of solutions 1 and 2 .

of dissolved ethanol) up to $\delta n_{\max }=5.4 \times 10^{-3}$ RIU (corresponding to $8.3 \times 10^{-2} \mathrm{w} / \mathrm{w}$ of dissolved ethanol). For each solution, the dip positions are monitored and determined from a centroid method ${ }^{1}$ based on finding the geometric center of the recorded dip $j$ as $x_{j}=\sum_{i} x_{i}\left(I_{\mathrm{th}}-I_{i}\right) / \sum_{i}\left(I_{\mathrm{th}}-I_{i}\right)$, where sole pixels $i$ with intensity values $I_{i}$ below a certain threshold $I_{\text {th }}$ are included in the centroid evaluation. In this work, a threshold corresponding to half the dip depth was chosen, and the resulting values $x_{j}$ for each dip have been averaged temporally over $N=10$ frames, yielding an averaged position value $\bar{x}_{j}$ of the dip.

In order to compensate for low-noise drift of the signal, each solution is referenced against pure water, in an exchange that leads to the temporal evolution of the dip position displayed in the inset of Fig. 3(b). This leads to the determination of refractive index differences by measuring associated spatial shifts $\delta x_{j}$ (measured in pixel units) of the SP resonance dips through $\delta x_{j}=(-1)^{j}\left(\bar{x}_{j}-2 \bar{x}_{j+1}+\bar{x}_{j+2}\right) / 2$. Each measured shift is then averaged over $M=10$ cycles, yielding resulting values $\langle\delta x\rangle$ associated with each $\delta n$. These values for all solutions are gathered in Fig. 4, on a logarithmic scale. Two sets of data are presented: one set obtained with an operating point $k_{y}$ close to the band gap (as in Figs. 3(a) and 3(b)) and for comparison, another set obtained faroff the band gap where the SP dispersion relation is getting very close to the one obtained over an unstructured planar metal film. The slope of each curve is extracted by fitting the intensity evolutions over the range where the response is well linear. Close to the M-point, the slope is measured as $\delta x / \delta n=43.0 \times 10^{3} \mathrm{px} / \mathrm{RIU}$ and is compared to $\delta x / \delta n$ $=18.8 \times 10^{3} \mathrm{px} /$ RIU in the planar film situation. These values reveal a more than twofold increase in sensitivity when resorting to the $1 \mathrm{PG}$.

At this stage, it is possible to evaluate the actual FOM of the $1 \mathrm{PG}$ sensor by measuring the dip widths close to the band gap. A measure of $w_{k_{y}}=98.1 \mathrm{px}$ leads to a $\mathrm{FOM}=438$ to be compared with the FOM $=228$ measured far away from the band gap (i.e., in the flat band region) where the width of the SP resonance has a value of 82.3 px. This increase in the FOM corresponds to a $90 \%$ improvement. We emphasize that this comparison is performed on the same 1PG and on the same image and therefore clearly assesses the relevance of exploiting the anisotropy of the plasmonic band structure in order to improve SP-based sensing figures of merit.

From a practical point of view, a clear estimate of the sensor resolution requires a baseline noise analysis of the setup. ${ }^{8}$ This was done by carefully evaluating the signal uncertainty (standard deviation) of the dip shift $\sigma_{\langle\delta x\rangle}$ over

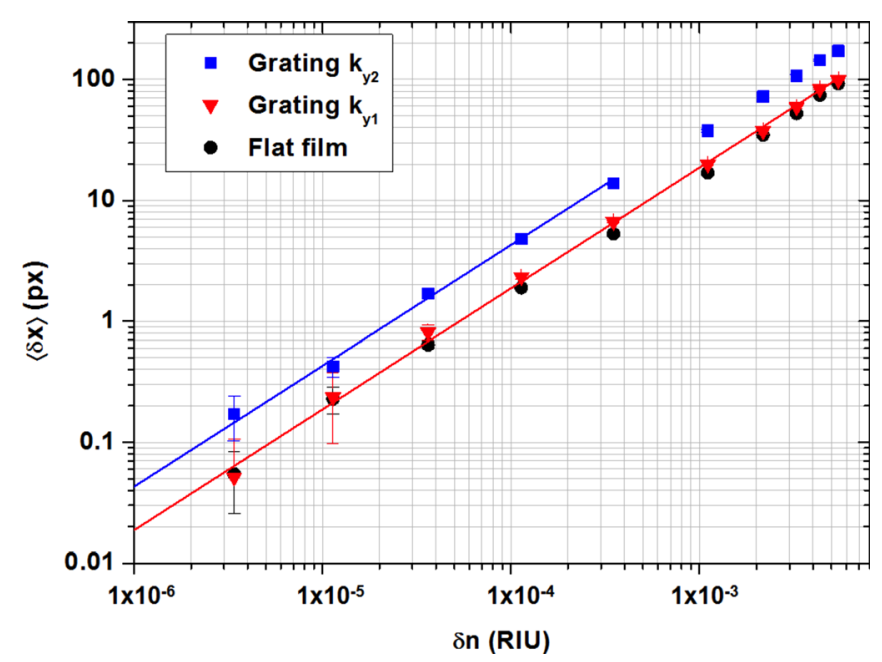

FIG. 4. Double logarithmic plot of the dip center variation $\langle\delta x\rangle$ as a function of the refractive index change $\delta n$ (averaged over 10 measurements). Results are shown close to the band gap (blue data points) and far-off from it, where the SP dispersion relation is the one of the flat surface (red data points). All data points have been taken from defocused Fourier space leakage radiation images. Results for $\delta n<10^{-3}$ RIU are fitted by linear regression, yielding a sensitivity of $\delta x / \delta n=43.0 \times 10^{3} \mathrm{px} / \mathrm{RIU}$ close to the band gap (at $k_{y}$ ) to be compared with the sensitivity of $\delta x / \delta n=18.8 \times 10^{3} \mathrm{px} / \mathrm{RIU}$ far-off it (see main text). Data from a flat gold film are also shown for comparison. As expected on a flat gold film, the sensitivity is lower with a linear fit yielding $16.6 \times 10^{3} \mathrm{px} / \mathrm{RIU}$. 
$3 \times 10^{5}$ frames. Accounting for the actual time $\tau=20 \mathrm{~s}$ necessary to exchange manually the liquid through the microfluidic cell and choosing to average over $N=10$ frames and $M=10$ cycles, an uncertainty of $\sigma_{\langle\delta x\rangle}=6.2 \times 10^{-2} \mathrm{px}$ was determined close to the band gap. This leads us to estimate a practical resolution of our sensor of $\sigma_{\langle\delta x\rangle} / S_{k}=1.4 \times 10^{-6}$ RIU. Neglecting the liquid exchange time increases even further the resolution which can be expected at $2.2 \times 10^{-7} \mathrm{RIU}$, i.e., comparable to the best systems reported to date. ${ }^{12}$ Throughout these analyses, a Faraday optical isolator was placed in the path of the illumination beam, preventing backreflections into the laser diode and thus increasing the whole stability of the source.

It is also interesting to note that the measured value of the FOM in the flat band region exceeds the one predicted theoretically from simulated band structures (shown in Fig. 2(b)) and calculated SP losses. By working with defocused images and thus dips instead of peaks, it is possible to saturate the detector around the resonance. This saturation is accompanied by a reduction of the dip width which turns out to be interesting to exploit in such a detection scheme, leading to an effective FOM improvement of the setup.

To conclude, we have demonstrated how the anisotropy of plasmonic band structure obtained at the level of plasmonic gratings can be exploited in a sensing context, turning out to enhance the actual resolution of SP-based sensors. We revealed a two-fold enhancement of the refractive index sensitivity using a leakage radiation microscope on which a specific defocusing strategy was adopted in order to better resolve the Fourier spatial shifts of the SP resonance as a function of refractive index changes. Our careful analysis demonstrated a resulting 90\% increase in sensing FOM. Having characterized the baseline noise of the whole system, we reached a sensing resolution as high as $O\left(10^{-6}\right)$ RIU. This remarkable value shows that our strategy can have a strong impact on future progress in the context of highresolution SP-based sensors. In particular, by exciting surface plasmons in different directions in different liquid cells, our scheme can be adapted to perform parallel sensing ${ }^{13}$ with such high resolution levels.

The authors acknowledge support from the ERC (Grant No. 227557) and the ANR (Equipex Union). B.S. acknowledges support from the Studienstiftung des deutschen Volkes and the Stiftung der deutschen Wirtschaft.

${ }^{1}$ J. Homola, Surface Plasmon Resonance Based Sensors (Springer-Verlag, Heidelberg, 2006).

${ }^{2}$ J. N. Anker, W. P. Hall, O. Lyandres, N. C. Shah, J. Zhao, and R. P. Van Duyne, Nature Mater. 7, 442 (2008).

${ }^{3}$ J. Homola, S. S. Yee, and G. Gauglitz, Sens. Actuators, B 54, 3 (1999).

${ }^{4}$ P. Zijlstra, P. M. R. Paulo, and M. Orrit, Nat. Nanotechnol. 7, 379 (2012).

${ }^{5}$ J. Feng, V. S. Siu, A. Roelke, V. Mehta, S. Y. Rhieu, G. Tayhas, R. Palmore, and D. Pacifici, Nano Lett. 12, 602 (2012).

${ }^{6}$ N. C. Lindquist, P. Nagpal, K. M. McPeak, D. J. Norris, and S.-H. Oh, Rep. Prog. Phys. 75, 036501 (2012).

${ }^{7}$ A. B. Dahlin, N. J. Wittenberg, F. Höök, and S.-H. Oh, Nanophotonics 2 , 83 (2013).

${ }^{8}$ B. Stein, J.-Y. Laluet, E. Devaux, C. Genet, and T. W. Ebbesen, J. Phys. Chem. C 116, 6092 (2012).

${ }^{9}$ C. J. Alleyne, A. G. Kirk, R. C. McPhedran, N.-A. P. Nicorovici, and D. Maystre, Opt. Express 15, 8163 (2007).

${ }^{10}$ B. Stein, J.-Y. Laluet, E. Devaux, C. Genet, and T. W. Ebbesen, Phys. Rev. Lett. 105, 266804 (2010).

${ }^{11}$ Because the grating is fabricated on ITO coated substrates, bonding of PDMS to glass by simple oxygen plasma activation could not be used. To achieve covalent bonding between PDMS and the gold surface, (3Mercaptopropyl)trimethoxysilane (Sigma-Aldrich) was applied from a $2 \%$ solution in methanol. The molecule provides a silane endgroup for binding to the PDMS mesh, as well as an opposing thiol group for bonding to the gold substrate.

${ }^{12}$ M. Piliarik and J. Homola, Opt. Express 17, 16505 (2009).

${ }^{13}$ N. C. Lindquist, A. Lesuffleur, H. Im, and S.-H. Oh, Lab Chip 9, 382 (2009). 